\title{
A case of invasive fungal sinusitis by candida species causing blindness
}

\author{
Subhramanyam $\mathrm{C}^{1, *}$, Mahesh Kumar Revoori ${ }^{1}$, Snehalatha Padiki ${ }^{1}$, Kashif Mohammed Mohiuddin ${ }^{2}, \operatorname{Kaul~}^{1}{ }^{1}$, Megha $S$ Uppin $^{3}$ and \\ Pranathi B ${ }^{4}$ \\ ${ }^{1}$ Department of Otorhinolaryngology, Krishna Institute of Medical Sciences, Secunderabad-500003, Telangana, India \\ ${ }^{2}$ Department of Pathology, Krishna Institute of Medical Sciences, Minister Road, Secunderabad-500003, Telangana, India \\ ${ }^{3}$ Department of Pathology, Nizam's Institute of Medical Sciences, Hyderabad - 500082, Telangana, India \\ ${ }^{4}$ Department of Ophthalmology, Krishna Institute of Medical Sciences, Secunderabad-500003, Telangana, India
}

\begin{abstract}
Invasive fungal sinusitis (IFS) is a common manifestation in immunocompromised patients. The common offenders are Aspergillus and Mucor. These fungal infections can cause various systemic complications, both intracranial and extracranial. Ultimately it may lead to fungemia and death. Very few cases of IFS caused by candida have been reported in the literature. Candida is a yeast like fungus, present normally as a commensal in the oral cavity and skin. Invasive fungal sinusitis with central retinal artery occlusion (CRAO) with candida is extremely rare. We are reporting a case of cavernous sinus thrombosis (CST) with CRAO causing bilateral blindness in a patient with invasive candida sinusitis.
\end{abstract}

Keywords: invasive fungal sinusitis; Candida; Aspergillus; fungal infections

*Corresponding author: Dr. C. Subhramanyam, MS., ENT (PGI, Chandigarh), Consultant surgeon, Department of Oto Rhino Laryngology, Krishna Institute of Medical Sciences (KIMS), Secunderabad-500003, Telangana, India. Email: drcsubrahmanyam@gmail.com

Received 9 September 2020; Revised 27 November 2020; Accepted 10 December 2020; Published 26 December 2020

Citation: Subhramanyam C, Revoori MK, Padiki S, Mohiuddin KM, Kaul S, Uppin MS, Pranathi B. A case of invasive fungal sinusitis by candida species causing blindness. J Med Sci Res. 2021; 9(1):4144. DOI: http://dx.doi.org/10.17727/JMSR.2021/9-7

Copyright: (C) 2021 Subhramanyam C et al. Published by KIMS Foundation and Research Center. This is an open-access article distributed under the terms of the Creative Commons Attribution License, which permits unrestricted use, distribution, and reproduction in any medium, provided the original author and source are credited.

\section{Introduction}

Central retinal artery occlusion (CRAO) is an ocular emergency. Patient typically presents with acute painless loss of vision which can be unilateral or bilateral. Being an ocular analogue of cerebral stroke, the most common risk factors are hypertension, diabetes and smoking. However, CRAO can also occur because of infiltration of CRA by fungi from the orbit. This usually happens in the setting of cavernous sinus thrombosis (CST), which is a serious condition manifesting with headache, swelling of eyeball, ptosis, restricted ocular movements, visual loss, fever and altered sensorium. The most common cause is underlying rhino-sinusitis $[1,2]$.

\section{Case report}

A 41-year-old gentleman, HbsAg +ve, diabetic, nonsmoker and non-alcoholic, presented with 10 days 
history of headache, fever, right facial swelling, nasal obstruction and running nose on a background of chronic sinusitis. As he developed ptosis, chemosis, and swelling of right eyeball in spite of a course of intravenous antibiotics, he was referred to us for further management.

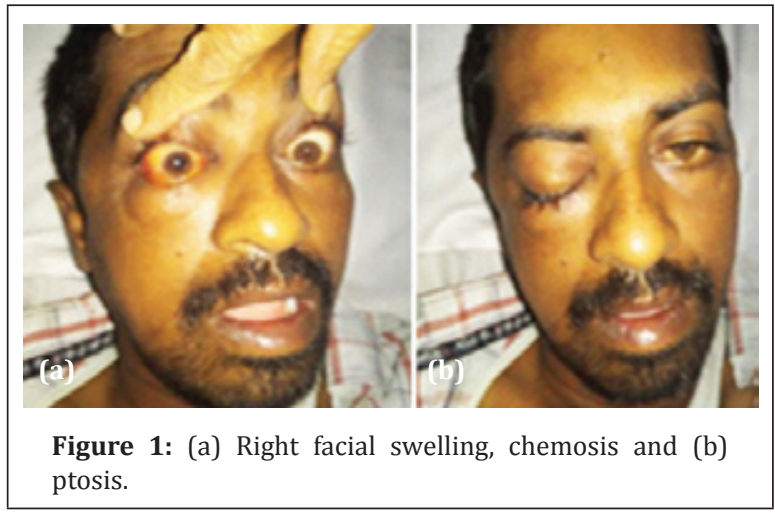

On examination, he had right facial swelling with difficulty in opening his mouth. Right eyelid was drooping with underlying congested eye ball and restricted eye movements (Figure 1) Vision was decreased, counting fingers was absent, but perception of light was present. Pupils were fixed and dilated. Left eye was normal. Nasal septum was deviated to left side with turbinate hypertrophy. A preliminary suspicion of invasive fungal sinusitis with CST was made. Patient was in sepsis at the time of admission as his serum c-reactive protein (CRP) was 27.98, procalcitonin was 40.9, WBC count was 12,000 and ESR was 68 . Patient was started on intravenous antibiotics. Interestingly the CT scan of paranasal sinuses done a week earlier showed haziness of left maxillary sinus, while the symptoms were on right side (Figure 2). MRI brain showed features suggestive of left IFS along with CST. There was also evidence of watershed infarcts in the territory of left Internal Carotid Artery (ICA) (Figure $3)$.

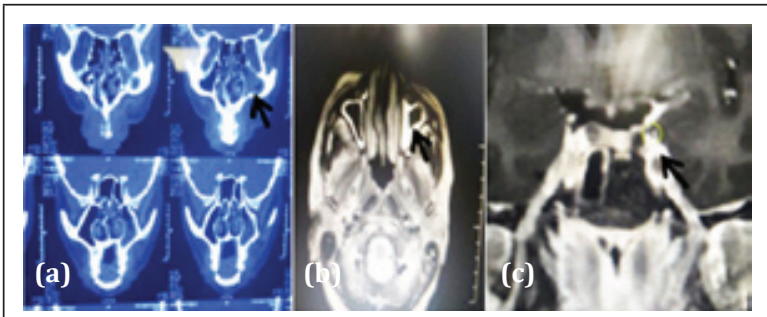

Figure 2a-c: CT, MR image shows left maxillary sinusitis along with CST.

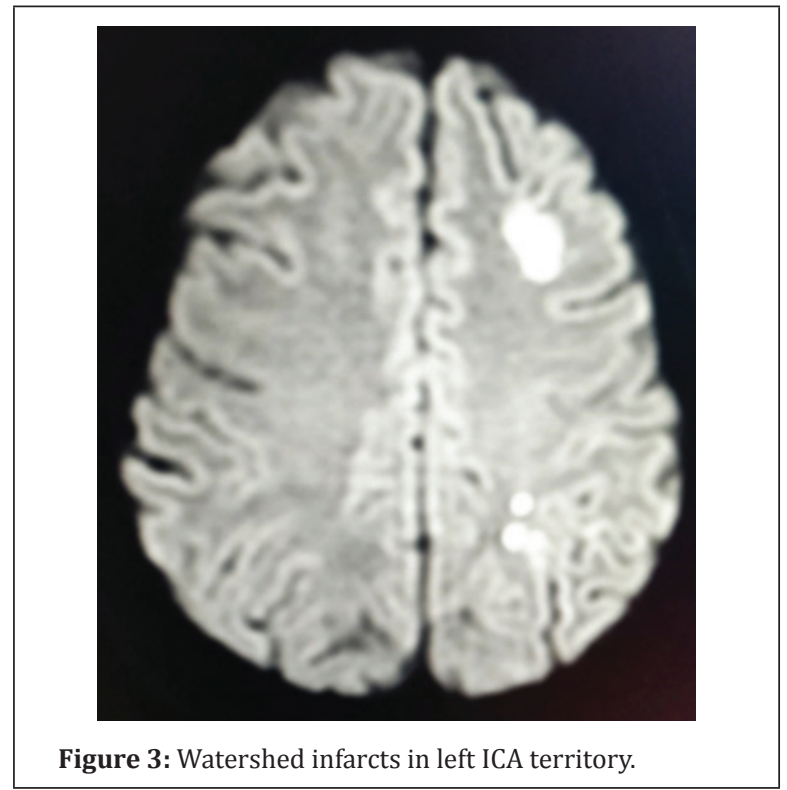

Within 24 hours of his admission in our hospital, patient rapidly progressed to develop proptosis, and chemosis in both eyes. Visual loss became bilateral with restricted ocular movements. Urgent FESS under local anesthesia revealed devitalized tissue in maxillarysinus.Frozenmountwasnegativeforfungus. Cerebrospinal fluid (CSF) examination revealed lymphocytic pleocytosis with normal proteins and sugar. Fungal and gram stains were negative for any organisms but showed occasional pus cells. Culture for fungal and bacterial organisms was negative, as was gene expert for MTB. Cryptococci Ag was also negative. However, given the combination of severe rhinosinusitis, CST and watershed infarcts in the left ICA territory, fungal etiology was strongly suspected and patient was empirically initiated on intravenous antifungal therapy. In spite of treatment he rapidly lost his vision bilaterally and his fundoscopy revealed pale opacified retina with box-carring appearance in both eyes suggestive of bilateral CRAO (Figure 4).

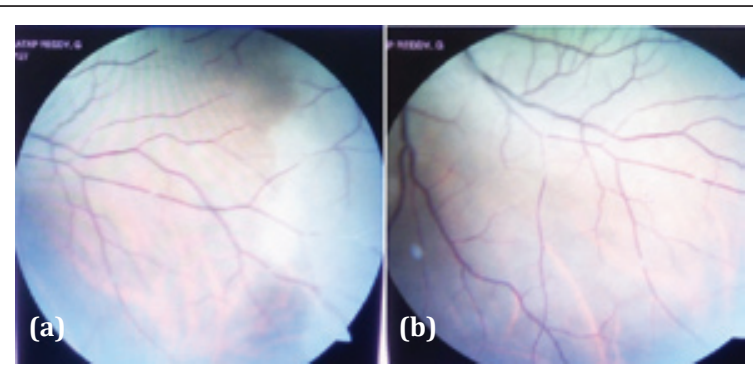

Figure 4a,b: Fundoscopy showing retinal opacification and box-carring appearance suggestive of bilateral retinal artery occlusion. 
Next day patient developed right hemiparesis due to left MCA infarction. Repeat CT PNS showed haziness in bilateral maxillary sinuses (Figure 5).

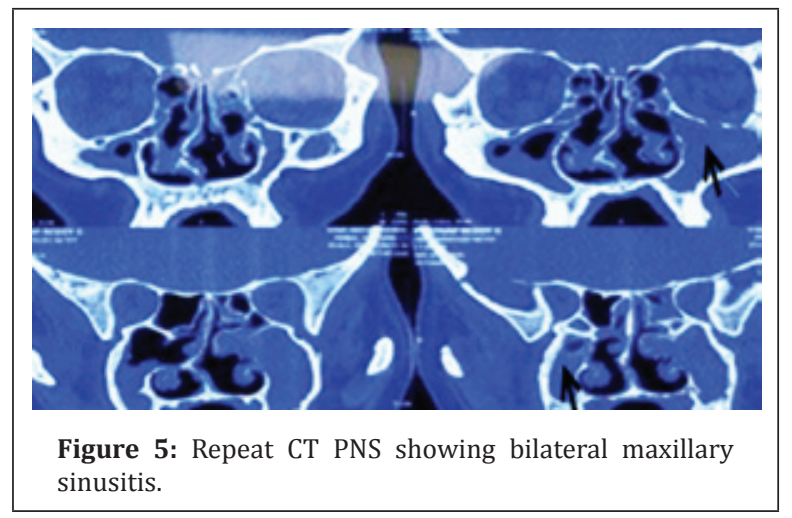

In view of relentless progression of symptoms, patient was taken up for revision FESS and debulking of necrotic debris from the sinuses. The frozen section revealed allergic mucin with no fungal elements on KOH mount. Gram stain showed plenty of pus cells with no organism. To our surprise histopathology revealed dense colonization by Candida organism with many yeast forms (Figure 6). Patient received liposomal amphotericin for a period of 3 weeks under close monitoring of renal function. Patient's neurological condition stabilized and while his vision did not recover, he could walk without support. He was advised to continue the treatment for a minimum of 6 months under closed supervision.

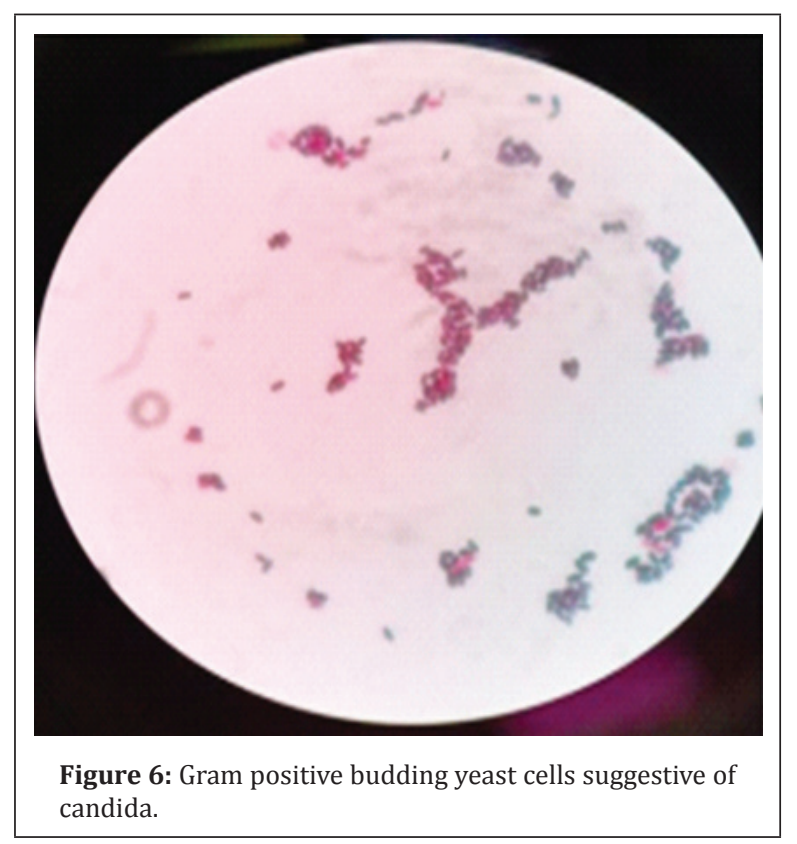

\section{Discussion}

The typical clinical picture of rhinosinusitis with cavernous sinus involvement associated with stroke or vascular occlusion is highly suggestive of a fungal etiology, particularly Aspergillus and Mucor [3, 4]. So, despite the non-identification of any organism in the first FESS biopsy sample, we put the patient on empirical antifungal treatment with liposomal Amphotericin. We persisted in our search by repeating the FESS after a week and were surprised by identification of Candida in the pathological sample, which was confirmed as Candida albicans on culture. Candida is a very uncommon cause of invasive fungal sinusitis and accounts for only 1-2\% of isolates [2, 5]. The reported species of Candida in Paranasal sinuses reported include Candida albicans [6, 7], Candida kefyr [8], Candida tropicalis and Candida kruesei [9]. It is important to identify the type of fungus to institute the most appropriate treatment to stop the direct, perineural and hematogenous spread of fungus. A sinusitis patient not responding to conventional antibiotic treatment should arouse the suspicion of a fungal etiology. Diagnostic nasal endoscopy, CT scan and repeated FESS procedures may be necessary in arriving at a diagnosis. The presumptive cause for tissue invasion and intracranial extension in our case was likely to be immunocompromised state because of HbsAg +ve status, diabetes and sepsis. Prolonged and indiscriminate use of antibiotics, steroid sprays and antihistamine agents in our patient of chronic sinusitis could also have contributed to fungal infections of paranasal sinuses because of suppression of normal bacterial flora.

\section{Conclusion}

Any patient of long standing chronic sinusitis can develop a superadded fulminant rhinosinusitis along with CST, bilateral CRAO and cerebral stroke within short period. Urgent and sometimes repeat biopsy is necessary to arrive at a correct diagnosis. Along with aspergillus and mucor, candida species should also be looked for as the causative agent.

\section{Conflicts of interest}

Authors declare no conflicts of interest. 


\section{References}

[1] Shah NJ, Rathore A. Intracranial extension of fungal sinusitis. Otorhinolaryngol Clin Int J. 2009; 1(1):55-61.

[2] Bassiri Jahromi S, Khaksar AA. Paranasal sinus mycosis in suspected fungal sinusitis. Iranian J Clin Infect Dis. 2006; $1(1): 25-29$.

[3] Michael RC, Michael JS, Ashbee RH, Mathews MS. Mycological profile of fungal sinusitis: an audit of specimens over a 7 -year period in a tertiary care hospital in Tamil Nadu. Indian J Pathol Microbiol. 2008; 51(4):493-496.

[4] Challa S, Uppin SG, Swetha Hanumanthu, Panigrahi MK, Purohit AK, et al. Fungal rhino sinusitis: A clinicopathological study from South India. Eur Arch Otorhinolaryngol. 2010; 267(8):1239-1245.

[5] Venkatachalam VP, Anand N. Paranasal mucormycosis: Unusual presentation in otherwise healthy child. Indian J Otolaryngol Head Neck Surg. 2007; 59(3):254-266.

[6] Panda NK, Sharma SC, Chakrabarti A, Mann SB. Paranasal sinus mycoses in north India. Mycoses. 1998; 41(7-8):281286.

[7] Chakraborti A, Sharma SC. Paranasal sinus mycoses. Indian J Chest Dis Allied Sci. 2000; 42(4):293-304.

[8] Joshi RR, Bhandary S, Khanal B, Singh RK. Fungal maxillary sinusitis: a prospective study in tertiary care hospital of eastern Nepal. Kathmandu Univ Med J. 2007; 5(2):195-198.

[9] Soontrapa P, Larbchaoensub N, Luxameechanporn T, Cheewaruangroj W, Prakunhungsit S, et al. Fungal rhinosinusitis: a retrospective analysis of clinicopathologic features and treatment outcomes at Ramathibodi hospital. Southeast Asian J Trop Med Public Health. 2010; 41(2):442449 . 